\title{
Impactos da pandemia do COVID-19 no desenvolvimento de crianças com o transtorno do espectro autista
}

\author{
Impacts of the COVID-19 pandemic on the development of children with autism spectrum \\ disorder
}

\author{
Impactos de la pandemia de COVID-19 en el desarrollo de niños con trastorno del \\ espectro autista
}

Amanda de Oliveira ${ }^{1 *}$, Isabela Galantini Silveira ${ }^{2}$, Isabela Soares Bôa Morte ${ }^{2}$, Jaqueline Maria de Azevedo Chagas $^{3}$, Juliana Tanaka Martins ${ }^{4}$, Marco Antonio Carvalho Gonçalves ${ }^{5}$, Maria Laura Pires de Carvalho Pereira ${ }^{2}$, Priscila Sabino dos Santos ${ }^{6}$, Talita Schmidt Bortoli ${ }^{7}$, Mônica Isaura Corrêa ${ }^{8}$.

\section{RESUMO}

Objetivo: Descrever os efeitos e discutir as consequências das medidas de contenção da COVID-19 em crianças com o Transtorno do Espectro Autista (TEA). Revisão bibliográfica: A pandemia mundial causada pelo coronavírus SARS-CoV-2 fez com que fossem necessárias medidas de restrição para evitar a circulação viral, incluindo o isolamento social, o uso de máscaras e cuidados de higiene. Essas medidas afetaram a vida de todas as pessoas, no entanto, portadores de doenças psiquiátricas foram especialmente afetados por esse contexto e crianças com TEA se mostraram vulneráveis às mudanças de rotina e medidas de distanciamento que a pandemia exigiu. Como essas crianças apresentam a necessidade de acompanhamento multidisciplinar e realização de atividades para desenvolvimento de habilidades, o fechamento de estabelecimentos, clínicas e escolas, bem como a mudança repentina de hábitos, afetaram a saúde mental e o desenvolvimento cognitivo, social e comportamental de crianças com o espectro autista. Considerações finais: Nesse contexto é perceptível a maior responsabilidade dos cuidadores dessas crianças em adaptar rotinas e atividades no ambiente domiciliar, a fim de prevenir maiores consequências, sendo necessário, também, o cuidado com o próprio bem-estar, de forma a evitar a sobrecarga e o estresse excessivo.

Palavras-chave: Transtorno do espectro autista, Infecções por coronavírus, Criança, Isolamento social.

\begin{abstract}
Objective: This article sought to analyze the literature about the impacts of the COVID-19 pandemic in kids with the Autism Spectrum Disorder. Bibliographic review: The worldwide pandemic caused by the coronavirus SARS-CoV-2 brought the necessity of restrictions to avoid viral circulation, including social isolations, use of masks and hygiene cares. These measures affected everybody's life but patients with psychiatric illness were especially affected by this context and children with ASD were vulnerable to the routine changes and distance measures that the pandemic required. Those children have the necessity of multidisciplinary monitoring and activities for skill development, the shutdown of establishments, clinics, and schools as well as the unexpected change of habits affected the mental health and the cognitive, social and behavioral development of kids with ASD. Final considerations: In this context, it is notable the increase of responsibilities of the caregivers to those kids in adapting routines and activities at the home environment, in order to prevent bigger consequences, on the other side is necessary to take care of themselves, to avoid the overload and excessive stress.
\end{abstract}

Key words: Autism spectrum disorder, Coronavirus infections, Child, Social isolation.

\footnotetext{
${ }^{1}$ Centro Universitário para o Desenvolvimento do Alto Vale do Itajaí (UNIDAVI), Rio do Sul - SC.

*E-mail: amanda.oliveira@unidavi.edu.br

${ }^{2}$ Centro Universitário de Belo Horizonte (UNIBH), Belo Horizonte - MG.

${ }^{3}$ Universidade de Rio Verde (UNIRV), Formosa - GO.

${ }^{4}$ Universidade Nove de Julho (UNINOVE), São Bernardo do Campo - SP.

${ }^{5}$ Fundação Educacional do município de Assis (FEMA), Assis - SP.

${ }^{6}$ Instituto de Medicina Integral Prof. Fernando Figueira (IMIP), Recife - PE.

7 Universidade Nove de Julho (UNINOVE), Osasco - SP.

${ }^{8}$ Faculdade de Medicina do Vale do Aço (UNIVAÇO), Ipatinga - MG.
}

SUBMETIDO EM: 5/2021 


\section{RESUMEN}

Objetivo: Este artículo pretende analizar el impacto de la pandemia de COVID19 en niños con Trastorno del Espectro Autista. Revisión bibliográfica: La pandemia mundial causada por el coronavirus SARS-Cov-2 significó que se necesitan medidas restrictivas para prevenir la circulación viral, incluyendo el aislamiento social, uso de máscaras y cuidados higiénicos. Estas medidas afectaron la vida de todas las personas, sin embargo, las personas con enfermedades psiquiátricas fueron especialmente afectadas, niños con TEA son vulnerables a los cambios de rutina y las medidas de distancia que la pandemia requiere. Estés ninõs tienen la necesidad de un seguimiento multidisciplinario y de actividades para desarrollar habilidades, el cierre de establecimientos, clínicas y escuelas así como el cambio repentino de hábitos, afectan la salud mental y el desarrollo cognitivo, social y conductual de ninõs con TEA. Consideraciones finales: En este contexto se nota la mayor responsabilidad de los cuidadores de estos niños en la adaptación de rutinas y actividades en sus casas, con el fin de prevenir mayores consecuencias, pero es necesario cuidar de su próprio bienestar para evitar sobrecargas y estrés excesivo.

Palabras clave: Trastorno del espectro autista, Infecciones por coronavirus, Ninõs, Aislamiento social.

\section{INTRODUÇÃO}

No ano de 2019, um novo coronavírus (SARS-CoV-2) passou a preocupar autoridades sanitárias do mundo todo quando se mostrou como agente etiológico de uma síndrome respiratória aguda grave denominada Coronavirus Disease 2019 (COVID-19). As infecções pelo novo coronavírus tiveram início na China, disseminando-se rapidamente ao redor do mundo e, devido à pandemia anunciada em março de 2020 pela Organização Mundial da Saúde (OMS), estamos vivenciando um dos momentos mais marcantes de toda a história. O mundo todo está passando por uma crise em diversos segmentos da sociedade, sendo os setores da saúde e da economia os mais afetados (CAVALCANTE JR, et al., 2020; WHO, 2020; PALACIO-ORTIZ JD, et al., 2020).

A principal forma de transmissão da COVID-19 ocorre através de gotículas respiratórias emitidas por pacientes infectados e por contato direto com os mesmos. Dessa forma, foram necessárias diversas medidas de restrição para evitar a circulação viral, incluindo o isolamento social, o uso de máscaras, cuidados de higiene e a alteração no funcionamento de diversos estabelecimentos, medidas essas que que mudaram o cotidiano de muitas pessoas (JIN Y, et al., 2020; AMORIM R, et al., 2020; CASAGRANDE M, et al., 2020).

Todas essas restrições, apesar de absolutamente necessárias e positivas para o controle da doença, acarretaram consequências psicológicas intensas em grande parcela da população. Pacientes portadores de doenças psiquiátricas foram especialmente afetados por esse contexto e, dentro desse grupo, crianças com Transtorno do Espectro Autista (TEA), se mostraram vulneráveis às mudanças de rotina e medidas de distanciamento que a pandemia exigiu (COX DJ, et al., 2020).

O TEA é um distúrbio do neurodesenvolvimento que se caracteriza por dificuldade na habilidade de interação social, elevada ansiedade, fácil distração e comportamentos estereotipados obsessivos e repetitivos. Ademais, pacientes com esse transtorno apresentam padrões restritos de interesses em atividades específicas, hiperatividade, e maior tendência à dependência da internet (AMERICAN PSYCHIATRIC ASSOCIATION, et al., 2014). O diagnóstico desse distúrbio aumentou significativamente nos últimos anos e, atualmente, uma a cada 54 crianças são diagnosticadas nos Estados Unidos (MAENNER MJ, et al., 2020).

Desde o início da pandemia, crianças com TEA foram particularmente afetadas pelas medidas de contenção da COVID-19, visto que necessitam de acompanhamento multidisciplinar de forma integral e constante. A necessidade do cuidado longitudinal justifica-se pela presença de níveis de ansiedade elevados, pela necessidade de rotinas estruturadas e pelas dificuldades de socialização características desses pacientes (KHOR AS, et al., 2014). Atividades que outrora as crianças realizavam de forma rotineira e presencial, passaram a ser estritamente virtuais, e várias outras foram suspensas. Aulas online e sem contato direto com os professores tornaram-se rotina, consultórios de psicólogos e terapeutas ocupacionais foram fechados e exercícios físicos ao ar livre impossibilitados. Nesse contexto, entende-se que a pandemia 
representou um desafio árduo para crianças com TEA e seus familiares, e demonstrou aumentar a irritabilidade, a inquietação e dificultar ainda mais a capacidade de concentração desses pacientes (PIZANO L, et al., 2020).

Dessa forma, o objetivo do presente artigo é descrever os efeitos e discutir as consequências das medidas de contenção da COVID-19 em crianças com o Transtorno do Espectro Autista, a partir da análise de produção científica atual sobre o tema.

\section{REVISÃO BIBLIOGRÁFICA}

O Transtorno do Espectro Autista (TEA) é um conjunto complexo de distúrbios que afetam o desenvolvimento neurológico e impactam a socialização do indivíduo. A primeira descrição do TEA na literatura foi feita pelo psiquiatra Leo Kanner, em 1943, através de uma série de casos contendo 11 crianças que apresentavam o que o autor descreveu à época como aparente nova forma de distúrbio emocional. Dentre as características percebidas por Kanner, ressalta-se obsessividade, estereotipia, ecolalia, dificuldade em estabelecer relacionamentos com novas pessoas e desejo de realizar suas atividades de forma rotineira e sozinhos (KANNER L, 1943; MANNING J, et al., 2020).

O entendimento atual do TEA é que se trata de um diagnóstico guarda-chuva, sob o qual amparam-se o Transtorno Autista, a Síndrome de Asperger, o Transtorno Desintegrativo da Infância e o Transtorno Generalizado do Desenvolvimento Não Especificado (SHANCHACK KE e THOMAS CA, 2016). Pessoas incluídas no espectro autista oscilam quanto à intensidade dos sintomas, que em geral, são: dificuldades na comunicação, na adaptação, na tomada de decisões, na interação social, no planejamento e direcionamento do comportamento; presença de comportamentos e interesses repetitivos, rígidos e incomuns, além de problemas em inibir impulsos, resultando em explosões de emoções (FERNANDES AA, et al., 2020; RODRIGUEZ IC e CORDERO AR, 2020).

Os sintomas podem surgir logo após o nascimento, com pico de incidência entre os 12 e 24 meses. O diagnóstico do transtorno é clínico, deve ser baseado em critérios pré-estabelecidos internacionalmente que requerem avaliação completa do desenvolvimento neuropsicomotor (DNPM) e costuma ser firmado entre os quatro e cinco anos de idade, o que frequentemente pode atrasar o início do tratamento e causar danos ainda mais graves ao DNPM da criança. A atenção dos pais às sutis manifestações e o acompanhamento pediátrico regular são cruciais para a identificação precoce deste e quaisquer outros transtornos que afetem negativamente o desenvolvimento infantil (LEMAY JF, et al., 2018; DIAS AA, et al., 2021).

A investigação inicia na busca por informações acerca da gestação, condições de parto da criança, possíveis fatores de risco e história familiar de transtornos do desenvolvimento (AMERICAN PSYCHIATRIC ASSOCIATION, 2014). Dentre as comorbidades associadas, podem estar incluídas alterações sensoriais e do sono, déficit intelectual e de linguagem, transtorno de ansiedade e epilepsia. Excluídos outros diagnósticos diferenciais, o exame físico pode reforçar a suspeita diagnóstica de TEA e deve incluir avaliação neurológica completa, além da observação do comportamento da criança e da sua interação com o meio e com seus familiares. A análise do DNPM por meio de testes é necessária para predizer se os desenvolvimentos físico, intelectual e linguístico da criança condizem com sua idade cronológica. Avaliações junto à fonoaudiologia podem contribuir para a precisão diagnóstica (SOCIEDADE BRASILEIRA DE PEDIATRIA, 2019).

Estimativas atuais apontam que 1,5\% da população mundial tem diagnóstico de TEA (THABTAH F, 2018). Nas últimas décadas, a incidência do TEA tem aumentado ao ponto em que no ano 2000, nos Estados Unidos, a prevalência era de um para 150 crianças, e em 2020 essa taxa alcançou um para cada 54 crianças (MANNING J, et al., 2020). Constata-se que parte desse aumento da prevalência é reflexo da evolução de testes diagnósticos e de rastreamento sensíveis, específicos e validados, sendo que o diagnóstico oportuno do TEA e o início rápido do tratamento são determinantes no prognóstico (DIAS AA, et al., 2021).

As causas do TEA não estão totalmente estabelecidas e continuam sendo estudadas. De acordo com Mandy W e Lay MC (2016), o desenvolvimento, seja ele típico ou atípico, tem origem nas relações e efeitos dinâmicos entre fatores genéticos e influências ambientais. Observou-se que a idade paterna elevada 
aumenta os riscos genéticos prévios, potencializando as chances de indivíduos vulneráveis geneticamente desenvolverem o fenótipo autista. Também, a idade materna superior a 35 anos significou risco 1,5 vezes maior de ter um filho dentro do espectro quando comparado a mulheres entre 25 e 29 anos de idade. Outros achados relevantes desse trabalho incluem associação positiva entre 0 uso de valproato na gestação e 0 nascimento de crianças pertencentes ao espectro autista e com outros distúrbios do desenvolvimento neurológico, bem como a poluição do ar relacionada ao tráfego comprovou-se fator de risco para o TEA.

Estudo transversal conduzido no Brasil em 2011 com 40 pais de crianças e adolescentes pertencentes ao espectro identificou, ao avaliar a escolaridade, que $77,5 \%$ dos pais possuíam o primeiro grau incompleto, enquanto que $22,5 \%$ das mães tinham o primeiro grau completo (OLIVEIRA AG, 2011). Em oposição, dados evidenciados por Berard M, et al. (2021) registram escolaridade de $51,46 \%$ dos pais e de $60,48 \%$ das mães correspondente ao ensino superior completo. A baixa renda familiar, profundamente impactada pelo nível escolar dos pais, é apontada pela Sociedade Brasileira de Pediatria (2019) como fator diretamente associado ao diagnóstico tardio e ao atraso do início das intervenções em crianças com TEA.

No que diz respeito ao tratamento dos pacientes com TEA, a presença da equipe multidisciplinar possibilita melhora cognitiva e comportamental do paciente. $O$ tratamento se baseia na estimulação do desenvolvimento de habilidades comportamentais e cognitivas que, se iniciadas precocemente e da forma correta, promovem a neuroplasticidade na criança e modificam o curso natural da doença (REIS e LENZA, 2020). Para isso, é necessário que todos os aspectos da vida do paciente estejam em conformidade com seu tratamento e que haja abordagem individual com pais e cuidadores para que eles saibam como estimular a criança corretamente e como lidar adequadamente com as mais diversas manifestações clínicas (VOLKMAR FR e WIESNER LA, 2019).

O estabelecimento de rotina de atividades, adequação nutricional, verificação de controle e qualidade do sono, bem como apoio familiar e escolar são preponderantes e complementares ao tratamento estabelecido pelo pediatra. Dessa forma, fica evidente a importância da estruturação familiar, bem como do trabalho interdisciplinar com psicólogos e fonoaudiólogos na obtenção de sucesso na evolução clínica do paciente com TEA (SOCIEDADE BRASILEIRA DE PEDIATRIA, 2019). Ainda que o TEA não possua cura, todo o cuidado à pessoa dentro do espectro visa auxiliá-la na formação de sua autonomia, de modo que esta possa apoiar sua família na manutenção de sua saúde e participar ativamente da comunidade na qual está inserida (FERNANDES AA, et al., 2020).

Atualmente, devido à alta transmissibilidade e transmissão comunitária da COVID-19 no Brasil, foram impostas medidas restritivas, visando reduzir o contato interpessoal nos principais locais de elevado fluxo de pessoas, como nos espaços de convívio comunitário, comércios, empresas, escolas, universidades, transportes públicos, eventos esportivos, culturais e outras situações que possam levar à aglomeração da população (OLIVEIRA WK, et al., 2020).

Ressalta-se que o lockdown, situação que envolve separação e restrição, é responsável por aflorar novos sintomas psiquiátricos, bem como desregular indivíduos que possuem diagnóstico de TEA anteriormente definido, seja pelo receio em torno da possibilidade de infecção pelo SARS-CoV-2, pelo seu estado de saúde geral, ou ainda, pelas mudanças no ambiente (BERARD M, et al., 2021; MARÍN JL, et al., 2021).

A descontinuidade na educação, no tratamento multidisciplinar e na rotina de atividades, inclusive físicas, representam importantes fontes de estresse nas crianças com diagnóstico de TEA. A baixa disponibilidade ou proibição do funcionamento presencial das instituições de suporte ao tratamento do TEA representou ruptura no desenvolvimento das crianças, que antes eram estimuladas com atividades periódicas e se viram impossibilitadas de praticar habilidades já adquiridas e até mesmo de aprender outras (GARCIA JM, et al., 2021; DIAS AA, et al., 2021).

É importante ressaltar que os desafios da educação infantil, mesmo antes da pandemia da COVID-19, englobavam o acolhimento às diversidades, com o intuito de arquitetar um conceito de educação capaz de acolher também as crianças portadoras de TEA de maneira individualizada. Esse acolhimento era planejado minuciosamente à luz de pedagogia específica e implantado através da associação de brincadeiras e planos 
educacionais, prática que ficou impossibilitada com a pandemia e que exigiu das escolas adaptações para que pudessem continuar promovendo interação das crianças na nova realidade do meio virtual (DIAS AA, et al., 2021).

Durante o processo de educação infantil são observados, por exemplo, a convivência, brincadeiras, participação, exploração e o autoconhecimento. Tais critérios são abordados por meio de metodologia pedagógica que possibilita às crianças experiências capazes de promover conhecimento de si mesmas e também do colega. Logo, é possível observar que durante o período pandêmico as crianças sofram com o isolamento social, evidenciando uma importante alteração na rotina diária que necessitou de adaptações para que, assim, as regressões das habilidades adquiridas não fossem tão contundentes (FERNANDES AA, et al., 2020).

Um estudo de coorte na França avaliou o impacto de medidas emergentes, como o lockdown, em 239 crianças e adolescentes com TEA. O estudo constatou que antes dessa medida de isolamento, $86 \%$ dos participantes frequentavam a escola, $61,4 \%$ tinham educação especial ou serviços de cuidados, $78,8 \%$ faziam acompanhamento com profissional, majoritariamente fonoaudiólogos, e apenas 5,5\% não recebiam nenhum tratamento. De modo geral, durante o lockdown, as intervenções foram mantidas por $75,8 \%$ dos participantes e descontinuadas por $24,2 \%$ destes. Os que tiveram continuidade nas intervenções, afirmaram ser sob a forma de entrevistas por telefone com os pais $(44,4 \%)$, videoconferências com a criança $(18,1 \%)$ ou consulta com a criança (apenas 8,6\%) (BERARD M, et al., 2021). Ressalta-se que portadores de TEA têm dificuldade em adaptar-se às mudanças, prezando sempre por seguir rotinas conhecidas, e a interrupção dos hábitos costuma despertar sentimentos de ansiedade e frustração, podendo ocorrer episódios de comportamento agressivo (LUGO-MARÍN J, et al., 2021).

Além do aumento da ansiedade e estresse na criança, esses sentimentos também são observados nos pais, que, com a nova doença, se viram cercados de preocupações sobre desemprego e renda familiar, aflição de alguém da família contrair o vírus e o receio da interrupção da terapia do filho. Até mesmo o home office, alternativa de empregadores para manutenção dos seus colaboradores nos respectivos postos de trabalho, modifica o estado psicológico dos cuidadores. O cuidado e a atenção aos filhos passou a ser integralmente realizado pelos pais, que neste período, tentaram instituir novas rotinas e horários na família para que a saúde mental das crianças fosse minimamente afetada (GARCIA JM, et al., 2021).

Outra dificuldade enfrentada neste período consistiu em encontrar a forma adequada para explicar às crianças com TEA o momento atual de crise sanitária que o mundo atravessa e o porquê da necessidade do uso de máscaras faciais e de se isolar em casa com a família. A adoção dessas recomendações tem fragilidades repousadas na dificuldade de a pessoa dentro do espectro não compreender a realidade. Por conta disso, instituições criaram manuais e guias com o propósito de ajudar os pais e familiares a contornar esta situação, fazendo o uso de técnicas que relatam, de maneira lúdica, a situação de saúde global atual, valendo-se de histórias curtas, com linguagem literal e ilustrações que representem o vírus. Somam-se a essas estratégias, a confecção e distribuição de panfletos que ensinam a lavagem correta das mãos e que contém a explicação do distanciamento social, para que assim as crianças possam compreender a mudança e colocar as medidas de segurança em prática (REICHER D, 2020).

Logo, famílias ao redor de todo o mundo precisaram se adaptar a essas mudanças (AMORIM R, et al., 2020). O momento da implementação das medidas restritivas pode acarretar danos na economia, além de baixa adesão da população com o passar do tempo. No entanto, caso o isolamento seja implementado de forma tardia, ou seja, realizado de maneira pouco eficaz, será incapaz de controlar o aparecimento de novos casos de COVID-19, situação que agrava a atual situação de acompanhamento e tratamento de crianças com TEA (OLIVEIRA WK, et al., 2020).

\section{CONSIDERAÇÕES FINAIS}

A partir do presente estudo, observou-se que, em se tratando de crianças e adolescentes com TEA, a adoção do isolamento social como forma de conter a contaminação do COVID-19 demonstrou desfechos 
desfavoráveis, quando considerados o desenvolvimento cognitivo, a estimulação da autonomia e espectro comportamental. Nesse contexto, é recomendado o estabelecimento de uma rotina, se possível com as atividades realizadas anteriormente de maneira remota, proporcionando a manutenção de habilidades já adquiridas e diminuindo as exacerbações das alterações comportamentais. Além disso, é de suma importância que os pais ou cuidadores forneçam informações simples e diretas, explicando sobre 0 distanciamento físico, as medidas de higiene e as modificações do dia a dia. Por fim, é fundamental que os pais e cuidadores dediquem tempo para o próprio bem-estar físico e emocional, para conseguirem enfrentar as adversidades impostas pela pandemia e oferecerem um ambiente acolhedor para as crianças durante esse período.

\section{REFERÊNCIAS}

1. AMERICAN PSYCHIATRIC ASSOCIATION. Manual diagnóstico e estatístico de transtornos mentais. 5 ed. Porto Alegre: Artmed, 2014; 992p.

2. AMORIM R, et al. The impact of COVID-19 on children with autism spectrum disorder. Revista de neurologia, 2020; 71(8): 285-291.

3. BERARD M, et al. Impact of containment and mitigation measures on children and youth with ASD during the COVID19 pandemic: Report from the ELENA cohort. Journal of Psychiatric Research, 2021; 137: 73-80.

4. CASAGRANDE M, et al. The enemy who sealed the world: effects quarantine due to the COVID-19 on sleep quality, anxiety, and psychological distress in the Italian population. Sleep Medicine, 2020; 75: 12-20.

5. CAVALCANTE JR, et al. COVID-19 no Brasil: evolução da epidemia até a semana epidemiológica 20 de 2020 . Revista Epidemiologia e Serviços de Saúde, 2020; 29(4): e2020376.

6. COX DJ, et al. A Proposed Process 260 for Risk Mitigation During the COVID-19 Pandemic. Behavior Analysis Practice, 2020; 13(2): 1-7.

7. DIAS AA, et al. Crianças com transtorno do espectro autista em tempos de pandemia: contextos de inclusão/exclusão na educação infantil. Zero-a-Seis, 2021; 23: 101-124.

8. FERNANDES AA, et al. Desafios cotidianos e possibilidades de cuidado às crianças e adolescentes com Transtorno do Espectro Autista (TEA) frente à COVID-19. Cadernos Brasileiros de Terapia Ocupacional, 2020; 29 : e2121.

9. GARCIA JM, et al. Brief report: The impact of the COVID-19 pandemic on health behaviors in adolescents with Autism Spectrum Disorder. Disability and Health Journal, 2021; 14(2): e101021.

10. JIN Y, et al. Virology, Epidemiology, Pathogenesis, and Control of COVID-19. Viruses, 2020; 12(4): 372.

11. KANNER L. Autistic disturbances of affective contact. Nervous Child, 1943; 2: 217-250.

12. KHOR AS, et al. Coping, Daily Hassles and Behavior and Emotional Problems in Adolescents with High-Functioning Autism/Asperger's Disorder. Journal of Autism and Developmental Disorders, 2014; 44: 593-608.

13. LEMAY JF, et al. Redesign of the autism spectrum screening and diagnostic process for children aged 12 to 36 months. Paediatrics \& child health, 2018; 5: 308-313.

14. LUGO-MARÍN J, et al. COVID-19 pandemic effects in people with Autism Spectrum Disorder and their caregivers: Evaluation of social distancing and lockdown impact on mental health and general status. Research in Autism Spectrum Disorders, 2021; 83: 101757.

15. MAENNER MJ, et al. Prevalence of autism spectrum disorder among children aged 8 years - autism and developmental disabilities monitoring network. Morbidity and Mortality Weekly Report: Surveillance Summaries, 2016; 69(4): 1-12.

16. MANDY W, LAI MC. Annual Research Review: The role of the environment in the developmental psychopathology of autism spectrum condition. Journal of Child Psychology and Psychiatry, 2016; 57(3): 271-92.

17. MANNING J, et al. Perceptions of Families of Individuals with Autism Spectrum Disorder during the COVID-19 Crisis. Journal of Autism and Developmental Disorders, 2020: 1-9.

18. NARZISI A. Handle the Autism Spectrum Condition during Coronavirus (COVID-19) Stay at Home Period: Ten Tips for Helping Parents and Caregivers of Young Children. Behavioral and Brain Sciences, 2020; 10(4): 207.

19. OLIVEIRA AG. Perfil do conhecimento dos pais de crianças autistas sobre o autismo. Trabalho de Conclusão de Curso (Pós-Graduação Lato Sensu em Neurociências) - Instituto de Ciências Biológicas, Universidade Federal de Minas Gerais, Belo Horizonte, 2011; 61 p.

20. OLIVEIRA WK, et al. Como o Brasil pode deter a COVID-19. Revista Epidemiologia e Serviços de Saúde, 2020; 29(2): e2020044.

21. PALACIO-ORTIZ JD, et al. Trastornos psiquiátricos en los niños y adolescentes en tiempo de la pandemia por COVID19, Revista Colombiana de Psiquiatría, 2020; 49(4): 279-288.

22. PISANO L, et al. A qualitative report on exploratory data on the possible emotional/behavioral correlates of Covid-19 lockdown in 4-10 years children in Italy. Society for the Improvement of Psychological Science and the Center for Open Science. 2020. Disponível em: https://psyarxiv.com/stwbn. Acessado em: 5 de abril de 2021.

23. RODRIGUEZ IC, CORDERO AR. Repercusión psicológica en niños con Trastorno del espectro autista durante el confinamiento por COVID-19. Multimed Revista Médica, 2020; 24(3): 690-707. 
24. REICHER D. Debate: Remote learning during COVID-19 for children with high functioning autism spectrum disorder. Child and Adolescent Mental Health, 2020; 25(4): 263-264.

25. REIS ST, LENZA N. A Importância de um diagnóstico precoce do autismo para um tratamento mais eficaz: uma revisão da literatura. Revista Atenas Higeia, 2020; 2(1):1-7.

26. SHANCHACK KE, THOMAS CA. Autism Spectrum Disorder: Primary Care Principles. American Family Physician, 2016; 94(12): 972-980.

27. SOCIEDADE BRASILEIRA DE PEDIATRIA. Manual de Orientação: Transtorno do Espectro do Autismo. 2019. Disponível em: https://www.sbp.com.br/fileadmin/user upload/21775d-MO _Transtorno_do_Espectro_do_Autismo_2_.pdf. Acessado em: 20 de março de 2021.

28. $\overline{T H A B T A H} \bar{F}$. An accessible and efficient autism screening method for behavioural data and predictive analyses. Health Informatics Journal, 2018; 25(4): 1-17.

29. VOLKMAR FR, WIESNER LA. Autismo: guia essencial para compreensão e tratamento. Porto Alegre: Artmed Editora, 2019; 349p.

30. WHO. Mental health and psychosocial considerations during the COVID-19 outbreak. 2020. Disponível em: https://www.who.int/docs/default-source/coronaviruse/mental-health-considerations.pdf. Acessado em: 18 de março de 2021. 